Autores:

Bautista, J.

Batalla, C.

Alfaro, $R$.

Research Group OPE-PROTHIUS. Dpto. de Organización de Empresas. Universitat Politècnica de Catalunya.
XX International Conference on Industrial Engineering and Operations Management

International IIE Conference 2014 23th-25th July, 2014 Málaga

\title{
ERgonomic Risk Minimization in Assembly Line Balancing
}

\section{INTRODUCTION. THE ENVIRONMENT}

Industrial plants with Mixed-Model Assembly Lines. Nissan powertrain plant in Barcelona.

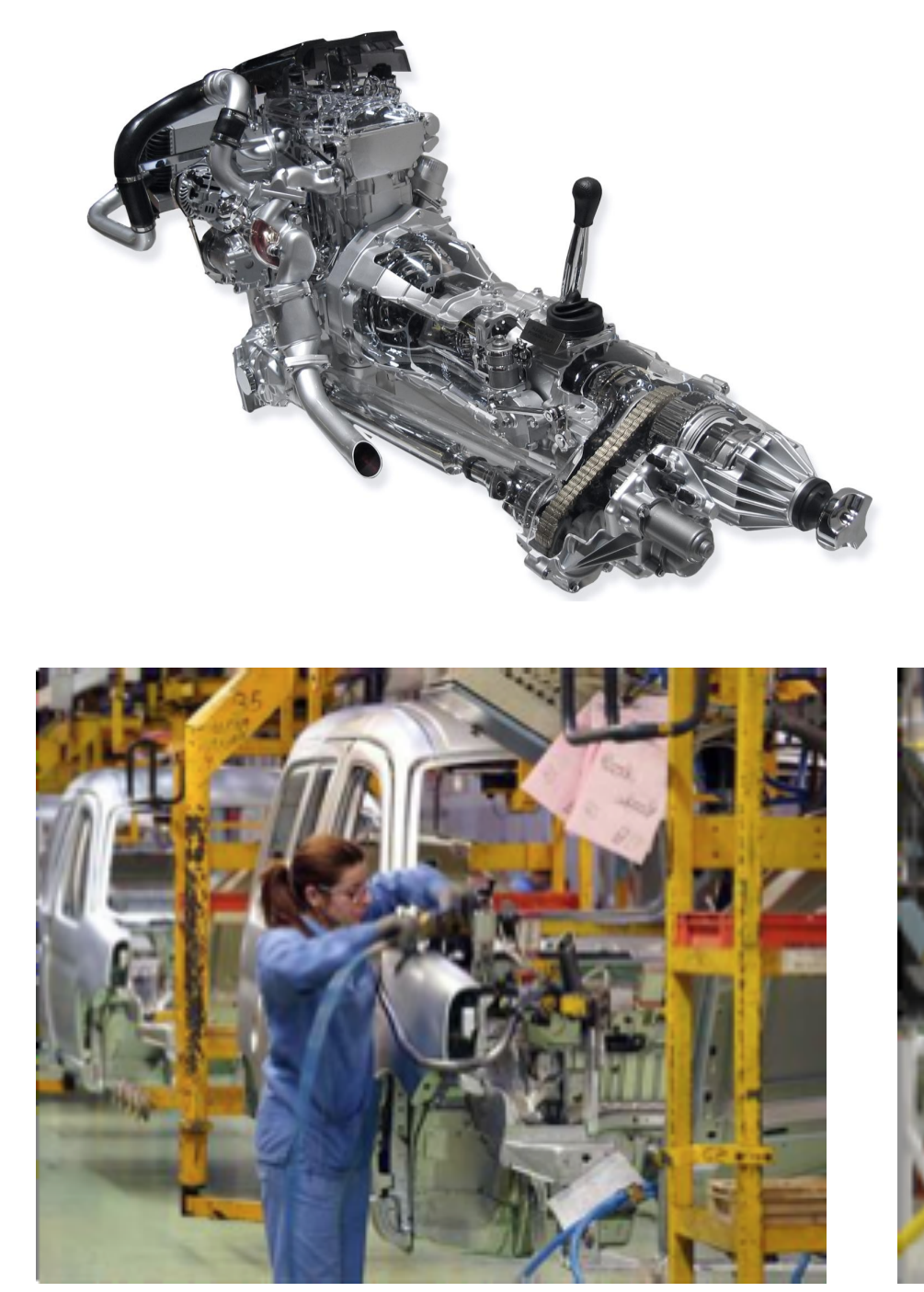

Repetitive movements

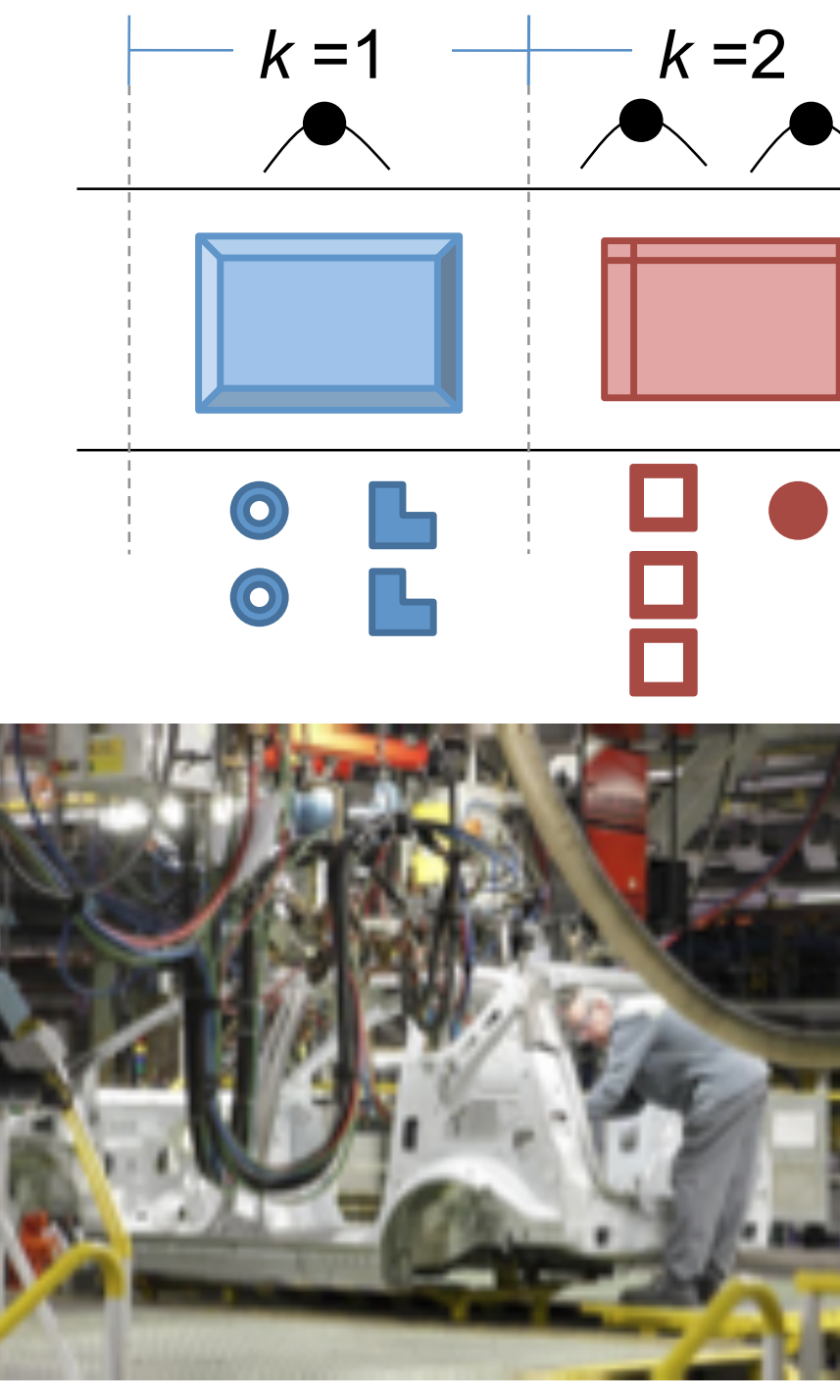

Postural loads

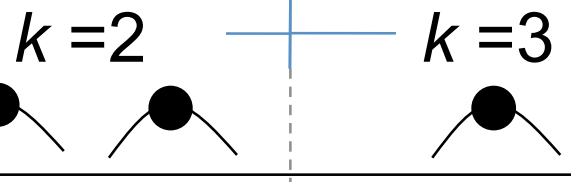
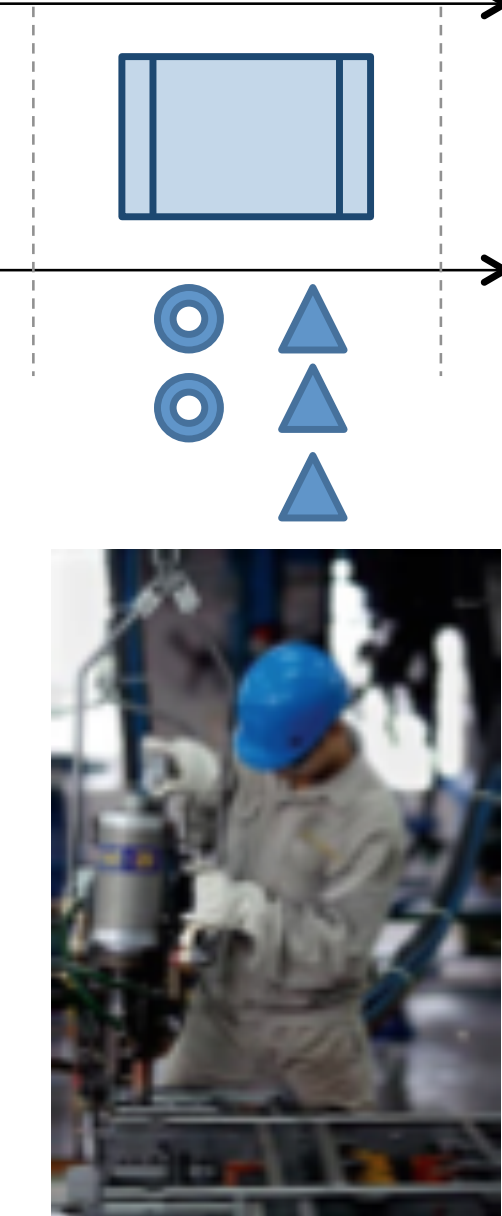

3. Assembly line Balancing Problems with Ergonomic Attributes

\section{Introduction. The Reference Problem}

Classic problem related to flow-oriented production systems: Assembly Line Balancing Problems (ALBP) Wokstation

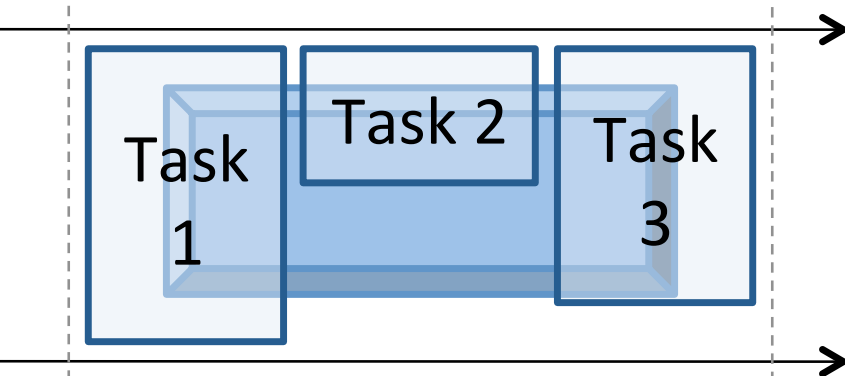

Given a set $J$ of elemental tasks or operations and a set $K$ of workstations, the assembly line balancing problem consist of establishing task assignments to the set of workstations satisfying the set of technological conditions that defines the order in which the operations must be realized (precedence constraints).
Contacto:

Joaquín Bautista Valhondo joaquin.bautista@upc.edu Avda. Diagonal, 647, 7th floor 08028 Barcelona, Spain 934011703

\section{Precedents works:}

- Rajabalipour et al., 2012 considered monotonous corporal postures, using the OWAS method (Ovako Working Analysis System) that only considers three corporal areas: back, arms and legs.

- Otto and Scholl, 2011 proposed two ways to consider the ergonomic risk into the SALBP (Simple Assembly Line Balancing Problem):

(1) Constraints that limit the maximum allowed ergonomic risk:

$$
F\left(S_{k}\right) \leq E r g, \forall k
$$

(2) A new objective function that minimizes the number of workstations and the global ergonomic risk of the line using a weighting coefficient:

$$
\min K^{\prime}(x)=K(x)+\omega \cdot \xi\left(F\left(S_{k}\right)\right)
$$

They evaluate the ergonomic risk with three methods: the NIOSH (National Institute for Occupational Safety and Health), the OCRA (Occupational Repetitive Action) and the EAWS (European Assembly Worksheet).

- Bautista et al., 2013 incorporated constraints that limit the minimum and maximum ergonomic risk to which workers may be exposed into the $T S A L B P$.

$$
F_{\phi}^{\min } \leq \sum_{j=1}^{|J|} F_{\phi, j} \cdot x_{j, k} \leq F_{\phi}^{\max }
$$

\section{Our proposal:}

- Minimize the maximum risk of line given, previously, the number of workstations, $m$, the cycle time, $c$, (at normal activity) and the available maximum area per workstation, $A$.

$J \quad$ Set of elemental tasks $(j=1, \ldots,|J|)$

$\Phi \quad$ Set of ergonomic risk factors $(\phi=1, \ldots,|\Phi|)$

$t$ Processing time required by the elemental $\operatorname{task}(j=1, \ldots,|J|)$

$a_{j} \quad$ Linear area required by the elemental $\operatorname{task}(j=1, \ldots,|J|)$

$X_{\phi, j}$ Category of the task $j$ associated to the risk factor $\phi$. Here it is a non-negative integer value between 1 and 4 .

$R_{\phi, j}$ Ergonomic risk of task $j$ associated to the risk factor $\phi$. Here $R_{\phi, j}=t_{j} \cdot X_{\phi, j}$

$P_{j} \quad$ Set of direct precedent tasks of the task $j$.

$m$ Number of workstations. In this case $m=|K|$

Variables:

$x_{j, k} \quad$ Binary variable equal to 1 if the elemental task $j$ is assigned to the workstation $k$, and to 0 otherwise.

$R_{\phi} \quad$ Maximum ergonomic risk associated to the risk factor $\phi$, and allowed to each workstation.
Other variants include more line attributes, such as: - The cycle time, $c$.

- The available lineal area for materials and tools on workstations, $A$.

Time and Space Assembly Line Balancing Problems (TSALBP)

Each task $j \in J$ requires an operation time $t_{j}$ and a lineal area $a_{j}$ and has a set $P_{j}$ of direct predecessors (problem precedence constraints). The TSALBP consist of grouping these tasks into $m$ stations minimizing the cycle time $c$, the number of workstations $m$, and/or the maximum available area for workstations $A$.

\section{Computational eXPerienCe}

$$
\begin{array}{ll}
\text { TSALBP-R_erg }: & \\
\qquad \begin{array}{l}
\min \bar{R}(\Phi)=\frac{1}{|\Phi|} \sum_{\phi=1}^{|\Phi|} R_{\phi} \\
\text { Subject to: }
\end{array} & (j=1, \ldots,|J|) \\
\sum_{k=1}^{|K|} x_{j, k}=1 & (k=1, \ldots,|K|) \\
\sum_{j=1}^{|J|} t_{j} \cdot x_{j, k} \leq c & (k=1, \ldots,|K|) \\
\sum_{j=1}^{|J|} a_{j} \cdot x_{j, k} \leq A & (k=1, \ldots,|K|) ;(\phi=1, \ldots,|\Phi|) \\
\sum_{j=1}^{|J|} R_{\phi, j} \cdot x_{j, k} \leq R_{\phi} & \left(1 \leq i, j \leq|J|: i \in P_{j}\right) \\
\sum_{k=1}^{|K|} k\left(x_{i, k}-x_{j, k}\right) \leq 0 & (j=1, \ldots,|J|) \\
\sum_{k=1}^{|K|} k \cdot x_{j, k} \leq m & (k=1, \ldots,|K|) \\
\sum_{j=1}^{J \mid} x_{j, k} \geq 1 & (j=1, \ldots,|J|) \wedge(k=1, \ldots,|K|) \\
x_{j, k} \in\{0,1\} &
\end{array}
$$

\section{Case study linked to an assembly line from Nissan's plant in Barcelona: \\ - Total demand of $T=270$ engines, divided into three types. \\ - 378 elementary tasks grouped into 140 operations. \\ - Cycle time equal to $180 \mathrm{~s}$. \\ - Available longitudinal area: $A \rightarrow \infty$

\begin{tabular}{|c|c|c|c|c|c|c|c|c|c|c|c|c|c|c|c|}
\hline \multirow{2}{*}{$k$} & \multicolumn{3}{|c|}{$|K|=19$} & \multicolumn{3}{|c|}{$|K|=20$} & \multicolumn{3}{|c|}{$|K|=21$} & \multicolumn{3}{|c|}{$|K|=22$} & \multicolumn{3}{|c|}{$|K|=23$} \\
\hline & $\frac{t_{k}}{100}$ & $a_{k}$ & $\begin{array}{l}R_{\theta, k} \\
\rho_{1}\end{array}$ & $\frac{t_{k}}{100}$ & $a_{k}$ & $R_{\phi, k}$ & $t_{k}$ & $a_{k}$ & $\begin{array}{l}R_{\phi, k} \\
\sigma_{1,2}\end{array}$ & $\frac{t_{k}}{171}$ & $a_{k}$ & $\begin{array}{ll}R_{0, k} \\
\Omega_{1,7}\end{array}$ & $\frac{t_{k}}{17}$ & $a_{k}$ & 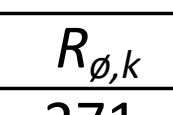 \\
\hline 1 & 180 & 7,5 & 265 & 180 & 6 & 300 & 180 & 6,5 & 285 & 171 & 8 & 267 & 173 & 7,5 & 271 \\
\hline 2 & 176 & 5 & 332 & 173 & 4,5 & 311 & 161 & 5 & 282 & 180 & 3 & 260 & 160 & 2,5 & 280 \\
\hline 3 & 179 & 5 & 298 & 180 & 6 & 280 & 175 & 4 & 290 & 142 & 4,5 & 284 & 168 & 5,5 & 276 \\
\hline 4 & 175 & 5 & 320 & 177 & 6,5 & 299 & 174 & 6,5 & 298 & 172 & 5,5 & 284 & 169 & 6 & 278 \\
\hline 5 & 125 & 7,5 & 350 & 105 & 6,5 & 315 & 105 & 5,5 & 290 & 105 & 4 & 275 & 110 & 3,5 & 280 \\
\hline 6 & 115 & 1,5 & 345 & 105 & 1,5 & 315 & 100 & 2,5 & 300 & 100 & 3,5 & 285 & 90 & 3,5 & 270 \\
\hline 7 & 115 & 1,5 & 345 & 105 & 2 & 315 & 100 & 2 & & 95 & 4 & 285 & 80 & 3,5 & 240 \\
\hline 8 & 120 & 1,5 & 340 & 105 & 0 & 315 & 100 & 1 & & 95 & 1,5 & 285 & 90 & 1 & 270 \\
\hline 10 & 年 & $\begin{array}{l}4,3 \\
55\end{array}$ & & 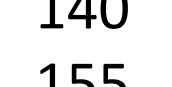 & 年, & & 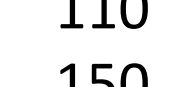 & & & 1250 & 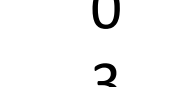 & & & & 270 \\
\hline 11 & 175 & 列 & 作 & 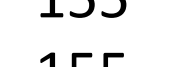 & , & & 年 & & & 年 & 1 & ( & & 2 & 280 \\
\hline 12 & 年 & 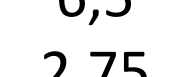 & & 年 & ז & & 150 & 年 & & 125 & 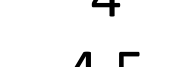 & 70 & & 隹 & 60 \\
\hline 13 & 100 & 15 & (A & 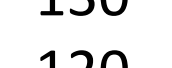 & 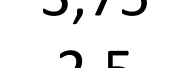 & & 110 & 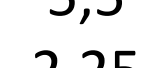 & & 100 & 年, & 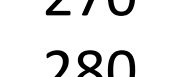 & & 然 & 60 \\
\hline 14 & & 3 & & 130 & 1 & & 10 & . & & 100 & 列 & & 110 & 列 & 80 \\
\hline & & & & 155 & 25 & & & 年 & & 120 & 25 & & 75 & 15 & 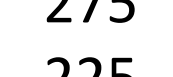 \\
\hline 16 & 180 & 4 & 345 & 170 & 3 & 315 & 145 & 3 & 295 & 125 & 1 & 285 & 120 & 0.5 & 270 \\
\hline 17 & 170 & 5 & 285 & 175 & 4,5 & & 140 & 1,5 & & 140 & & 85 & 130 & 2,5 & 265 \\
\hline 18 & 160 & 4,25 & 265 & 180 & 4,5 & 5 & 175 & & & 140 & 1,5 & 30 & 140 & 3 & 80 \\
\hline 19 & 180 & 2 & 250 & 170 & 3,75 & 295 & 165 & 4 & 5 & 140 & & & 160 & 2,5 & 80 \\
\hline 20 & & & & & 3 & & 175 & 4,75 & 290 & 170 & 4,5 & 80 & 150 & 3,5 & 75 \\
\hline 21 & & & & & & & & & & 180 & 4,25 & 80 & 165 & & 280 \\
\hline 22 & & & & & & & & & & 180 & 2,5 & 255 & 115 & 2,75 & 225 \\
\hline & 157,4 & 4,0 & 323,4 & 149,5 & 3,8 & 307,3 & 142,4 & 3,6 & 292,6 & 135,9 & 3,4 & 279,3 & 130,0 & 3,3 & 267,2 \\
\hline & & 15 & 250 & $10^{\circ}$ & 0 & & & & & & & 255 & 75 & 0,5 & 225 \\
\hline & 180 & 7,5 & 350 & 180 & 6,5 & 315 & 180 & 6,5 & 300 & 180 & & 285 & 180 & 7,5 & 280 \\
\hline
\end{tabular} \\ - Several values for the number of workstations: $m=\{19,20,21,22$ and 23$\}$. \\ - One ergonomic risk factor (physical). The methods used to evaluate the ergonomic risk have been: the RULA, OCRA and NIOSH method, respectively, by modifying the levels of risk of each method on a common scale from 1 (acceptable risk) to 4 (unacceptable risk). \\ - Solver CPLEX v11.0. \\ 5. CONCLUSIONS}

-We have proposed a line balancing model with the aim of minimizing ergonomic risk and compliance with the temporary and spatial restrictions.

- Through a case study from Nissan, we have proposed a progressive reduction of the risk category of the line (which is translated into operators with fewer injuries) through the creation of new jobs without changing the production capacity of the plant.

6. ACKNOWLEDGMENT

This work is supported by the Spanish Ministerio de Educación y Ciencia under Project DPI2010-16759 (PROTHIUS-III) including EDRF fundings. 